\title{
Downregulation of LRIG1 expression by RNA interference promotes the aggressive properties of glioma cells via EGFR/Akt/c-Myc activation
}

\author{
RUIFAN XIE*, HAI YANG*, QUNGEN XIAO, FENG MAO, SUOJUN ZHANG, FEI YE, \\ FENG WAN, BAOFENG WANG, TING LEI and DONGSHENG GUO
}

\begin{abstract}
Department of Neurosurgery and Sino-German Neuro-Oncology Molecular Laboratory, Tongji Hospital, Tongji Medical College, Huazhong University of Science and Technology, Wuhan, Hubei 430030, P.R. China
\end{abstract}

Received September 4, 2012; Accepted October 11, 2012

DOI: $10.3892 /$ or.2012.2102

\begin{abstract}
The LRIG1 [leucine-rich repeats and immunoglobulin-like domains (LRIG)] gene is not universally downregulated in human cancers, and its role in tumorigenesis and the development of glioma has not been well addressed. In this study, we used short hairpin RNA (shRNA)-triggered RNA interference (RNAi) to block LRIG1 gene expression in the GL15 human glioma cell line. Specific downregulation of LRIG1 by shRNA resulted in significantly enhanced capabilities of proliferation, inhibition of apoptosis and invasion in the GL15 cells. LRIG1 repression induced marked activation of epidermal growth factor receptor (EGFR), protein kinase B (Akt) and c-Myc signaling molecules. Our results demonstrated that RNAi against LRIG1 may effectively downregulate LRIG1 gene expression. LRIG1 functions as a tumor suppressor in the pathogenesis of glioma via EGFR/Akt/c-Myc activation.
\end{abstract}

Correspondence to: Dr Baofeng Wang, Department of Neurosurgery and Sino-German Neuro-Oncology Molecular Laboratory, Tongji Hospital, Tongji Medical College, Huazhong University of Science and Technology, Wuhan, Hubei 430030, P.R. China

E-mail: wangbaofeng@yahoo.cn

*Contributed equally

Abbreviations: LRIG, leucine-rich repeats and immunoglobulinlike domains; shRNA, short hairpin RNA; RNAi, RNA interference; EGFR, epidermal growth factor receptor; GBM, glioblastoma multiforme; GAPDH, glyceraldehydes-3-phosphate dehydrogenase; ECM, extracellular matrix; MTT, 3-(4,5-dimethylthiazol-2-yl)2,5-diphenyltetrazolium; DMSO, dimethyl sulfoxide; OD, optical density; PI, proliferation index; MMP, matrix metalloproteinase

Key words: leucine-rich repeats and immunoglobulin-like domains 1, glioma, RNA interference, proliferation, invasion, epidermal growth factor receptor

\section{Introduction}

Glioblastoma multiforme (GBM), the most frequently encountered primary malignant tumor of the central nervous system, has an extremely poor prognosis in spite of treatments including surgery, radiotherapy, chemotherapy and immunotherapy. GBM is characterized by uncontrolled cell growth and diffused infiltration of adjacent normal brain tissues, making complete surgical resection virtually impossible and relapse inevitable. The 2-year survival rate of GBM patients is no more than $27 \%$ even for those patients treated with radiotherapy plus temozolomide (1). Therefore, there is an urgent need for developing novel therapeutic strategies for glioblastomas. Currently, gene therapy is considered a new possible approach.

The human leucine-rich repeats and immunoglobulin-like domains (LRIG) gene family consists of 3 homologous genes, LRIG1, LRIG2 and LRIG3, which were observed to be widely expressed in human tissues $(2,3)$. The first identified member in this family, the LRIG1 gene, located at chromosomal band 3 p14, a common region where homozygous deletions often occur in several types of tumors, demonstrated high expression in the brain relative to other tissues (4-6). When compared with the corresponding normal tissues, LRIG1 expression appeared reduced or even absent in several types of tumors (7-10). It has been reported that LRIG1 enhanced the ubiquitylation and degradation of epidermal growth factor receptor (EGFR) and was involved as a negative feedback attenuator of the EGFR-mediated signaling (11). The LRIG1 gene has been proposed as a tumor suppressor and a prognostic predictor in several types of tumors (12-14). To date, a survey of public data demonstrates that LRIG1 gene expression does not reveal a general downregulation in human tumors. For example, the overexpression of the LRIG1 gene has been observed in prostate cancers $(15,16)$, leukemia and astrocytoma (17) compared to the corresponding normal tissues.

As to the effect of LRIG1 on glioblastoma, our previous study demonstrated that the upregulation of LRIG1 expression induced apoptosis and suppressed the growth of glioma cells (18). Moreover, we discovered that the perinuclear localization of the LRIG1 protein in astrocytic tumors was associated with low WHO grade and better survival of the patients (19). 
The effect of LRIG1 knockdown on the malignant properties of glioma cells and the underlying mechanism has yet to be reported. In this study, we designed two short hairpin RNA (shRNA) plasmids targeting the LRIG1 gene and successfully transfected them into a human glioblastoma cell line, GL15. The transfected cells demonstrated a markedly decreased expression levels of LRIG1 mRNA and protein. The specific and effective downregulation of LRIG1 resulted in a significantly increased proliferative rate, decreased apoptosis and increased invasive capability of GL15 cells. We also explored the possible underlying mechanisms of the effects of LRIG1 knockdown. We demonstrated that LRIG1 downregulation notably increased the activation of EGFR, AKT and c-Myc. These results demonstrated that LRIG1 downregulation promoted the malignant properties of glioma cells by enhancing the activation of EGFR/Akt/c-Myc and led to the proposal that LRIG1 may act as a tumor-suppressor gene in glioblastoma.

\section{Materials and methods}

Cell line and culture. The human glioblastoma GL15 cell line was kindly donated by Dr Håkan Hedman (Umeå University Hospital). GL15 cells were maintained in Dulbecco's modified Eagle's medium (DMEM) supplemented with $10 \%(\mathrm{v} / \mathrm{v})$ fetal bovine serum (FBS) (Hyclone, Logan, UT, USA) under a humidified atmosphere of $5 \% \mathrm{CO}_{2}$ at $37^{\circ} \mathrm{C}$. The medium was replaced normally every 3 days. Cells were passaged every 5 or 6 days and routinely examined.

Vector-based plamid construction for short hairpin RNAs. The full-length sequence of human LRIG1 mRNA was obtained from Genbank (accession: NM_015541)(4). Short interfering RNAs (siRNAs) targeting against LRIG1 were designed by means of a web server (http://jura.wi.mit.edu/ bioc/siRNA) (20) and according to the principle of Tuschl (21). Two target sequences were selected from the screening results and synthesized as documented in Table I. One unspecific sequence with no homology to any human-derived gene was also synthesized as the negative control to provide a baseline for experiments. The structure of the oligonucleotides is Bam HI + sense chain + loop + antisense chain + termination signal + SalI + HindIII. The hairpin siRNA inserts were separately ligated into BamHI-HindIII linearized pGenesil-2 vector (Genesil Corp., Wuhan, China) according to the manufacturer's instructions. All the inserted sequences were verified by DNA sequencing. Each constructed plasmid contains the neomycin/kanamycin-resistance gene to enable the selection of kanamycin-resistant colonies in bacteria and G418-resistant clones in mammalian cells. The RNA interference (RNAi) plasmid DNAs for LRIG1 and the negative control were then prepared for cell transfection.

Stable transfection of GL15 cells with pGenesil2-LRIG1 shRNA. GL15 cells were seeded on 6-well culture plates at $3 \times 10^{5}$ cell/well, maintained in DMEM containing $10 \%(\mathrm{v} / \mathrm{v})$ FBS and grown to $85-95 \%$ confluency. Metafectene transfection agents (Biontex, Munich, Germany) were then used to perform the transfection strictly according to the manufacturer's instructions. After $48 \mathrm{~h}$ of transfection, the medium was replaced with complete medium containing $600 \mu \mathrm{g} / \mathrm{ml}$ G418 (Amresco, Solon, Ohio, USA). After 2 weeks of selection, the G418-resistant clones that represented possible stably transfected cells were individually selected and expanded for further experiments.

Quantitative reverse transcription-polymerase chain reaction $(q R T-P C R)$. Total RNAs from cultured cells were isolated using TRIzol reagent (Invitrogen, Carlsbad, CA, USA) according to the manufacturer's instructions. After the amounts of total RNA were determined by ultraviolet (UV) spectrophotometry, $1 \mu \mathrm{g}$ of total RNA was used as a template for reverse transcription using ReverTra Ace-A (Toyobo, Osaka, Japan). Quantitative real-time PCR analysis was performed with SYBR-Green PCR Master Mix (Toyobo) according to the manufacturer's protocol. DNA primer sequences of LRIG1 were designed as follows: sense, 5'GGT GAG CCT GGC CTT ATG TGA ATA3' and antisense, 5'CAC CAC CAT CCT GCA CCT CC 3'. The realtime PCR program was used as follows: $50^{\circ} \mathrm{C}$ for $2 \mathrm{~min}, 95^{\circ} \mathrm{C}$ for $2 \mathrm{~min}$, followed by 35 cycles of $95^{\circ} \mathrm{C}$ for $15 \mathrm{sec}, 60^{\circ} \mathrm{C}$ for $30 \mathrm{sec}, 72^{\circ} \mathrm{C}$ for $45 \mathrm{sec}$ and $72^{\circ} \mathrm{C}$ for $10 \mathrm{~min}$. Each sample was tested in triplicate and each real-time PCR experiment included a positive and negative control. The relative gene expression was quantified by Applied Biosystems and compared using the $\mathrm{Ct}$ method. The mRNA levels of the target gene $\left(2^{-\Delta \Delta C t}\right)$ were normalized to the endogenous 18 -sec method reference $(\Delta \Delta \mathrm{Ct})$ and related to the amount of target RNA in the control sample, which was set at 1.0 on the calibrator.

Western blotting and immunoprecipitation. Cells were scraped into pre-cold RIPA buffer (Beyotime Biotech, Nantong, China) for $10 \mathrm{~min}$. All subsequent manipulations were performed on ice. The supernatant containing the proteins was collected after centrifugation. The protein concentrations were determined with a BCA protein assay kit (Beyotime Biotech). After being mixed with $5 \mathrm{X}$ loading buffer, the protein samples were subjected to heat-denaturation at $100^{\circ} \mathrm{C}$ for $5 \mathrm{~min}$. Then the protein $(70 \mu \mathrm{g})$ of each sample was loaded onto $8 \%$ SDS-PAGE gels for electrophoresis and transferred to nitrocellulose membranes, which were blocked with TTBS (Tween-Trisbuffered saline) containing 5\% non-fat milk at room temperature for $1 \mathrm{~h}$ to prevent non-specific binding. Diluted polyclonal rabbit anti-LRIG1 (1:1,000; Abcam, Cambridge, MA, USA), monoclonal mouse anti-GAPDH and polyclonal rabbit anti-cMyc (1:1,000; Santa Cruz Biotechnology, Inc., Santa Cruz, CA, USA), polyclonal rabbit anti-phospho-EGFR and anti-EGFR (1:1,000; Upstate Biotech, Lake Placid, NY, USA), polyclonal rabbit anti-phospho-ERK, anti-ERK, anti-phospho-AKT and anti-AKT antibodies (1:1,000; Cell Signaling Technology, Danvers, MA, USA) were added and incubation was carried out overnight at $4^{\circ} \mathrm{C}$, respectively. The membranes were then washed with TTBS 3 times and incubated with the 1:3,000 diluted corresponding secondary antibodies (goat anti-rabbit $\mathrm{IgG}$, goat anti-mouse IgG; ProteinTech, Chicago, IL, USA) at $37^{\circ} \mathrm{C}$ for $1 \mathrm{~h}$. After being washed with TTBS for 3 times, the protein and antibody conjugations were visualized using a DAB detection system. Images were captured and analyzed by Quantity One software. For the immunoprecipitation experiment, $1 \mathrm{mg}$ of cell lysates obtained through NP-40 lysis buffer (Beyotime Biotech) was subjected to precipitation with $2 \mu \mathrm{g}$ of 
Table I. Oligonucleotide sequences of LRIG1-specific siRNA.

Name

siRNA sequences $\left(5^{\prime} \rightarrow 3^{\prime}\right)$
Target nucleotide sites on LRIG1 cDNA
pGenesil2-negative control

pGenesil2-LRIG1 siRNA1

pGenesil2-LRIG1 siRNA2

\author{
ACTACCGTTGTTATAGGTG \\ ACTCTCTGAGATTGACCCT \\ GGCCTACCTTTCCTTAGAA
}

249-267

$420-438$
anti-EGFR primary antibody and protein A Sepharose CL-4B (GE Healthcare, Waukesha, WI, USA). Precipitates were then assayed using western blotting as previously described.

MTT assay. The proliferation rates of LRIG1-siRNA1 and control cells were measured by 3-(4,5-dimethylthiazol2-yl)-2,5-diphenyltetrazolium bromide (MTT) assay. Cells were seeded at a density of $5 \times 10^{3} /$ well in 96 -well plates and maintained in complete culture medium containing $300 \mu \mathrm{g} / \mathrm{ml}$ G418 for 0, 3, 6, 8 and 10 days. MTT (Sigma-Aldrich, St. Louis, MO, USA) was then added to the medium at a final concentration of $0.5 \mathrm{mg} / \mathrm{ml}$. After $4 \mathrm{~h}$ of incubation, cells of each well were dissolved in $150 \mu 1$ dimethyl sulfoxide (DMSO) (Sigma-Aldrich). The optical density (OD) was measured using ELISA-type plate reader equipment at a wavelength of $490 \mathrm{~nm}$ with a baseline subtraction reading. Each time point was repeated 6 times.

Cell cycle analysis by flow cytometry. The distribution of different cell cycle phases (G0/G1, S or G2/M phase) is characterized by DNA content, which is reflected by varying fluorescent intensities of propidium iodide, a DNA binding fluorescence dye. In this experiment, cells were synchronized by serum starvation for $24 \mathrm{~h}$, and then incubated with complete medium for $48 \mathrm{~h}$. Cells were harvested with trypsin-EDTA, washed with chilled PBS twice and fixed with $70 \%$ ethanol at $4^{\circ} \mathrm{C}$ overnight. The fixed cells were collected, re-suspended in $400 \mu \mathrm{l}$ PBS containing $50 \mu \mathrm{g} / \mathrm{ml}$ propidium iodide and $50 \mu \mathrm{g} / \mathrm{ml}$ Rnase A (Sigma-Aldrich) for $30 \mathrm{~min}$ at $37^{\circ} \mathrm{C}$ in the dark. Cells $\left(1 \times 10^{6}\right)$ for each sample were analyzed using a FACScalibur II sorter and Cell Quest FACS system (BD Biosciences, Franklin, NJ, USA). The proliferation index $(\mathrm{PI})=[(\mathrm{S}+\mathrm{G} 2 / \mathrm{M}) /(\mathrm{G} 0 / \mathrm{G} 1+\mathrm{S}+\mathrm{G} 2 / \mathrm{M})]$, was calculated according to the percentage of cells in different phases. The experiment was performed in triplicate and the results were averaged.

Annexin V-FITC/propidium iodide double labeling for FCM-assessed apoptosis. The extent of spontaneous apoptosis was determined with an Annexin V-FITC/propidium iodide kit (KeyGEN Biotech, Nanjing, China) according to the manufacturer's instructions. Cells were synchronized by serum starvation, and then incubated with complete medium for $48 \mathrm{~h}$. The cells were harvested through trypsinization, centrifuged at 1,000 rpm and the pellet was re-suspended in $1 \mathrm{X}$ binding buffer at a density of $1 \times 10^{6}$ cells $/ \mathrm{ml}$. The prepared suspensions $(100 \mu \mathrm{l})$ for each sample were incubated with $5 \mu \mathrm{l}$ of FITC-conjugated Annexin V and $10 \mu \mathrm{l}$ of propidium iodide for $15 \mathrm{~min}$ at room temperature in the dark. Another $400 \mu \mathrm{l}$ of $1 \mathrm{X}$ binding buffer was added to each sample before analysis.
FACScalibur II sorter and Cell Quest Research Software were used as previously described. The experiment was performed in triplicate.

Cell invasion assay. The invasive capability of GL15 cells in vitro was measured by Transwell chamber assay. Diluted ECM (Sigma-Aldrich) gel solution $(50 \mu \mathrm{l})$ was added to the upper chamber of the Transwell insert $(6.5 \mathrm{~mm}, 8-\mu \mathrm{m}$ pore size; Costar Inc.). The inserts were incubated at $37^{\circ} \mathrm{C}$ for $4 \mathrm{~h}$ for gelling and were pretreated with serum-free DMEM medium at $37^{\circ} \mathrm{C}$ for $1 \mathrm{~h}$ before seeding cells at a density of $2 \times 10^{4}$ cells/well in $100 \mu 1$ medium with $1 \%$ FBS. The lower chambers were filled with $500 \mu \mathrm{l}$ DMEM containing $10 \%$ FBS. After $24 \mathrm{~h}$ of incubation at $37^{\circ} \mathrm{C}$ with $5 \% \mathrm{CO}_{2}$, the cells on the upper side of the insert filter were removed with a cotton swab and cells that had invaded through the ECM-coated filter were fixed in $10 \%$ methanol and stained with trypan blue. The number of invaded cells was counted under a light microscope at a magnification of $\mathrm{x} 200$ in 5 predetermined fields. The experiment was performed in triplicate.

Gelatin zymography. Equal numbers of cells (2x10 $/$ well) were seeded in 6-well plates and grown in media containing $10 \%$ FBS for $24 \mathrm{~h}$. The media were then replaced with fresh DMEM without serum. After incubation for $24 \mathrm{~h}$, the conditioned media were harvested and subjected to the analysis for gelatinolytic activities of matrix metalloproteinase-2 (MMP-2) and MMP-9. Briefly, equal amounts of conditioned media were separated by $10 \%$ SDS-polyacrylamide gels impregnated with $0.1 \%$ gelatin. The gels were washed twice with $2.5 \%$ Triton X-100 for $45 \mathrm{~min}$ and incubated with zymogram developing buffer at $37^{\circ} \mathrm{C}$ for $\sim 48 \mathrm{~h}$. The gels were then stained with a staining solution $(0.5 \%$ Coomassie Blue, $45 \%$ methanol and $10 \%$ acetic acid) for $3 \mathrm{~h}$, and destained in $45 \%$ methanol and $10 \%$ acetic acid. Zones of gelatinolytic activity were detected as clear bands against a blue background.

Statistical analysis. The data are expressed as the means \pm standard deviation (SD). Statistical analyses were performed using SPSS statistical software (SPSS Inc.). For group comparison the Student's t-test followed by a least significant difference t-test (LSD) were used. $\mathrm{P}<0.05$ was considered to indicate a statistically significant difference.

\section{Results}

The vector expressing LRIG1 shRNA causes specific and effective downregulation of LRIGl expression. Two shRNAexpressing plasmids (siRNA1 and siRNA2) and a negative control plasmid were constructed and stably transfected into 
Table II. Effects of LRIG1 downregulation on the distribution of cell cycle phases of GL15 cells.

\begin{tabular}{lccccc}
\hline Groups & Samples & G0/G1 $(\%)$ & S $(\%)$ & G2/M (\%) & PI $(\%)$ \\
\hline Control & 4 & $77.59 \pm 4.13$ & $15.22 \pm 3.71$ & $7.19 \pm 1.39$ & $22.41 \pm 4.13$ \\
siRNA1 & 4 & $28.79 \pm 4.00^{\mathrm{a}}$ & $10.23 \pm 2.04^{\mathrm{b}}$ & $60.98 \pm 5.22^{\mathrm{b}}$ & $71.21 \pm 4.00^{\mathrm{b}}$ \\
\hline
\end{tabular}

Data are presented as the means $\pm \mathrm{SD},{ }^{\mathrm{a}} \mathrm{P}<0.05,{ }^{\mathrm{b}} \mathrm{P}<0.01$. PI, proliferation index.
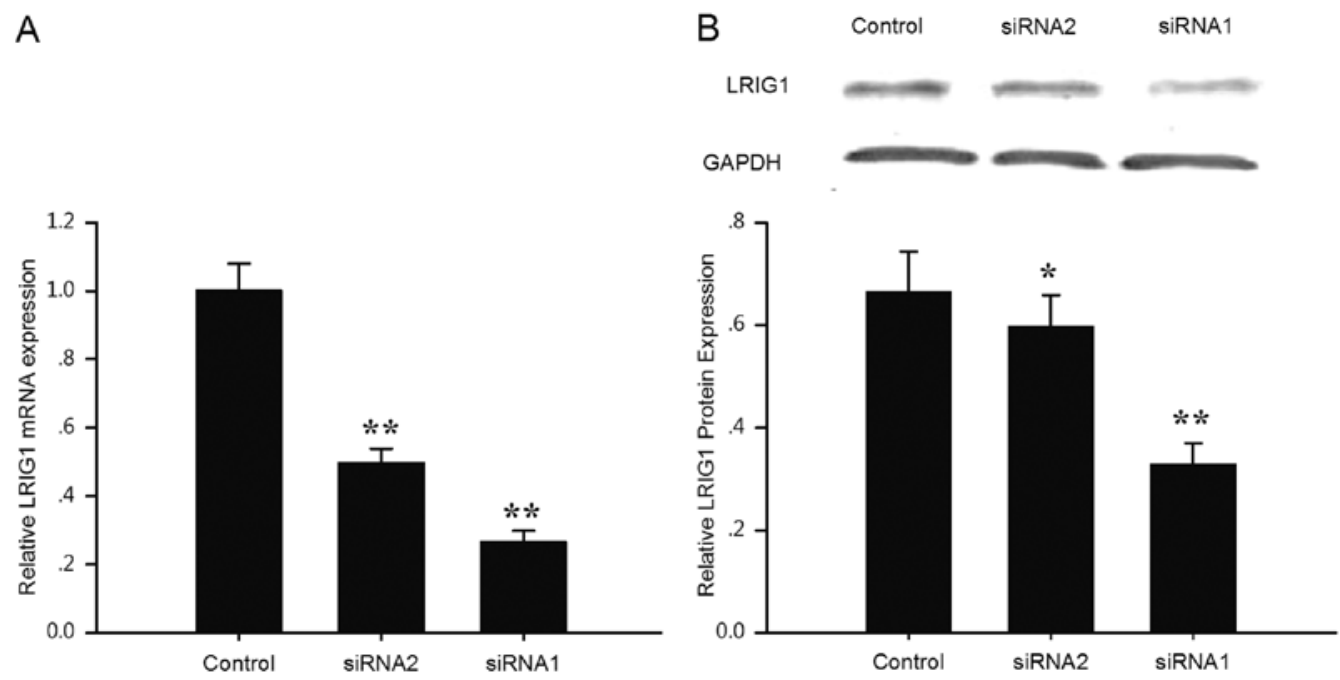

Figure 1. LRIG1 expression was effectively downregulated by vector-based RNA interference. (A) GL15 cells were transfected with different LRIG1-siRNA and negative control constructs. LRIG1 mRNA was quantified by real-time RCR. The LRIG1 mRNA was significantly inhibited by the LRIG1 siRNA1 construct. (B) The protein levels of LRIG1 were measured by western blotting and semi-quantitative data are displayed in the lower panel. Compared with the control, the protein levels of LRIG1 in the siRNA1- and siRNA2-transfected GL15 cells were reduced by 50.5 and 10.1\%, respectively. Data are presented as the means $\pm \mathrm{SD}\left({ }^{*} \mathrm{P}<0.05,{ }^{* *} \mathrm{P}<0.01\right)$.

GL15 cells. The LRIG1 and housekeeping gene, glyceraldehyde-3-phosphate dehydrogenase (GAPDH), mRNA and protein levels were measured by quantitative real-time PCR and western blotting, respectively (Fig. 1). Compared with the negative control cells, LRIG1 transcripts were reduced by 73.5 and $50.4 \%$ in the siRNA1- and siRNA2-transfected cells, respectively (Fig. 1A). In line with the real-time PCR results, the expression level of the LRIG1 protein was reduced by 50.5 and $10.1 \%$ in the siRNA1 and siRNA 2 cells, respectively (Fig. 1B). The results indicated that the expression of LRIG1 was downregulated specifically and effectively by LRIG1 shRNA and that siRNA1 exhibited a stronger knockdown ability compared to siRNA2. Thus the LRIG1-siRNA1 transfected cells were expanded for further study.

Effects of LRIG1 silencing on cell proliferation. Having established the effective LRIG1-specific knockdown transfectant, we used MTT assay to examine th e cell proliferation of the transfected cells. The proliferation rate of GL15 cells stably expressing siRNA1 was significantly higher compared to that of the negative control cells (Fig. 2A).

To investigate whether LRIG1 promotes cell proliferation by regulating the cell cycle progression, fluorescence-activated cell sorting following propidium iodide staining was performed (Fig. 2B). The number of cells in the G2/M phase significantly increased in the LRIG1-siRNA1 cells compared to the nega- tive control cells. The PI was calculated and a marked increase in PI in LRIG1-siRNA1 cells was observed (Table II). The data demonstrated that LRIG1 knockdown resulted in an increase of cell proliferation in GL15 cells.

Effects of LRIG1 silencing on cell apoptosis. To determine the extent of spontaneous apoptosis, we analyzed the apoptosis in the LRIG1-siRNA1 and control cells by double staining with Annexin V-FITC and propidium iodide via flow cytometry. The percentages of apoptotic cells were $7.05 \pm 1.45$ and $13.66 \pm 2.36 \%$ in the LRIG1-siRNA1 and negative control cells, respectively $(\mathrm{P}<0.01)$ (Fig. 3$)$. The level of spontaneous apoptosis was significantly decreased in the LRIG1-siRNA1 cells. The percentage of cells that accumulated in the lower left quadrant was much higher as compared with the negative control cells, indicating that the number of normal proliferative cells was increased compared with that of the control cells, which was in accordance with the results of the cell cycle analysis by flow cytometry.

Effect of the downregulation of LRIGI on the invasive capability of the GL15 cells. Invasive growth pattern is a distinct characteristic feature of glioblastoma and prevents total tumor resection. ECM gel-coated Transwell chamber assay was used to investigate the effect of the knockdown of LRIG1 on invasive capability. The number of invading cells was $158.2 \pm 20.0$ in the 
A
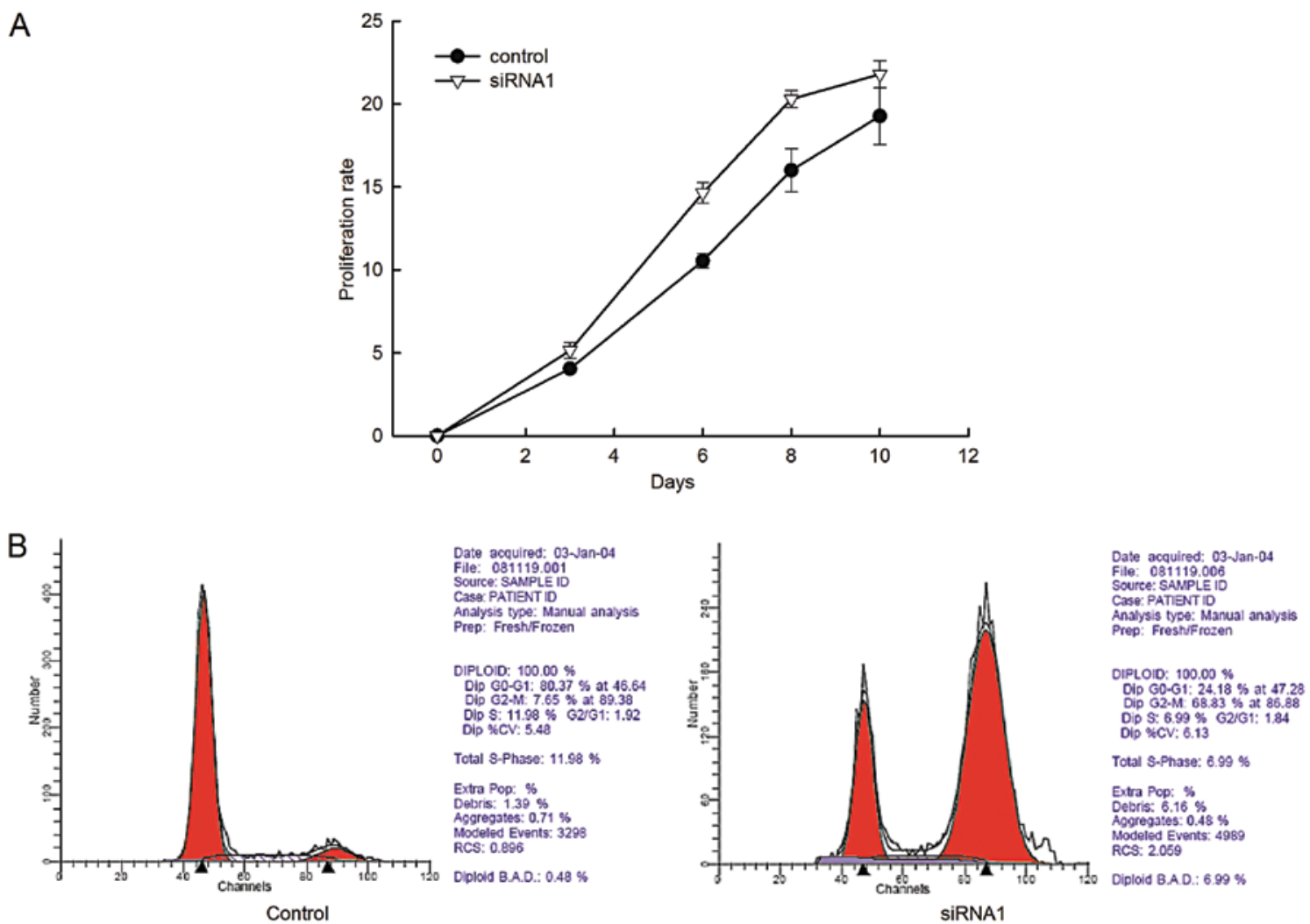

Figure 2. Effect of LRIG1 knockdown on cell proliferation and the cell cycle. (A) The proliferative capability of GL15 cells (LRIG1-siRNA1 and the control) were measured by MTT assay at the indicated times. The growth rate of LRIG1-siRNA1 cells was markedly higher compared to that of the negative control cells. Data are presented as the means \pm SD. (B) To further confirm the effect of LRIG1 on cell growth, cell cycle distribution was analyzed by FACS. The number of cells in the G2/M phase was significantly increased in the LRIG1-siRNA1 targeted cell group compared with the negative control.

A

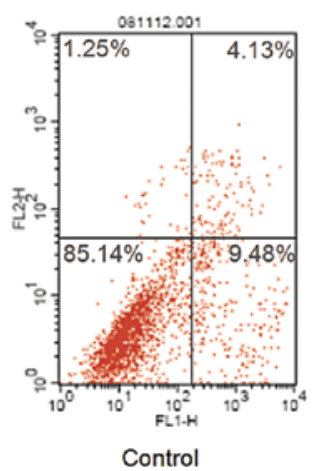

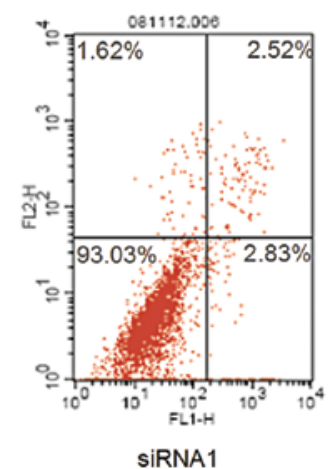

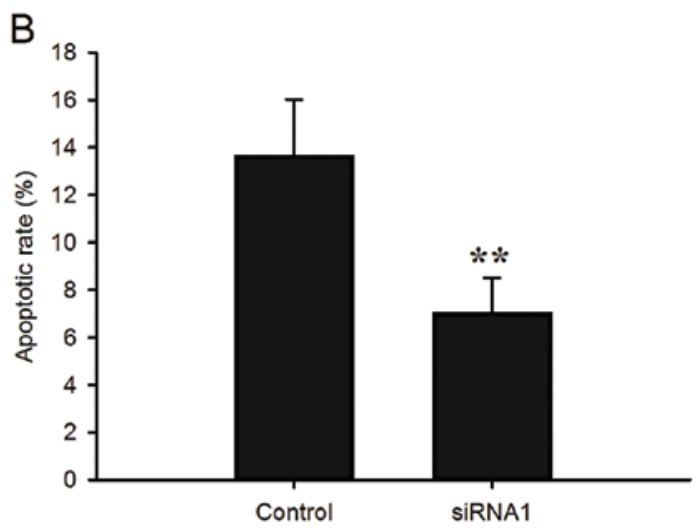

Figure 3. Effect of the downregulation of LRIG1 on the apoptotic rate of GL15 cells. (A) Representative flow cytogram of Annexin V binding vs. propidium iodide uptake is shown. (B) Total apoptosis (early apoptosis plus late apoptosis) of LRIGl-siRNA and control cells is presented. The total apoptotic rates were $7.05 \pm 1.45$ and $13.66 \pm 2.36 \%$ in the LRIG1-siRNA1 and control cell groups, respectively, which demonstrated that LRIG1-siRNA1 decreased apoptosis in GL15 cells. Data are presented as the means $\pm \mathrm{SD}$ of 4 experiments. $\left({ }^{* * *} \mathrm{P}<0.01\right)$.

LRIG1-siRNA1 cells and 65.3 \pm 5.4 in the negative control cells (Fig. 4A and B). The downregulation of LRIG1 significantly increased the invasive capability of the GL15 cells.

The MMPs are closely related to the invasive capability. Using gelatin zymography, we further examined the gelatinolytic activity of MMPs in LRIG1-siRNA1 cells. The results revealed that the levels of MMP-2 and MMP-9 expression were both significantly increased in LRIG1-siRNA1 cells in comparison to the control cells (Fig. 4C). These data indicated that LRIG1 knockdown enhanced the invasive capacity of GL15 cells.
LRIG1 silencing promotes the activation of EGFR, AKT and $c-M y c$. Previous studies have demonstrated that LRIG1 forms a protein complex with EGFR in several non-glioma cell lines. In the present study, using the co-immunoprecipitation experiment, we demonstrated that LRIG1 also forms a specific protein complex with endogenous EGFR in the GL15 cell line (Fig. 5A).

In this study, we further investigated the effect of LRIG1siRNA1 on the EGFR-mediated downstream signaling pathways of PI3K/Akt and MAPK/ERK. When stimulated 
A

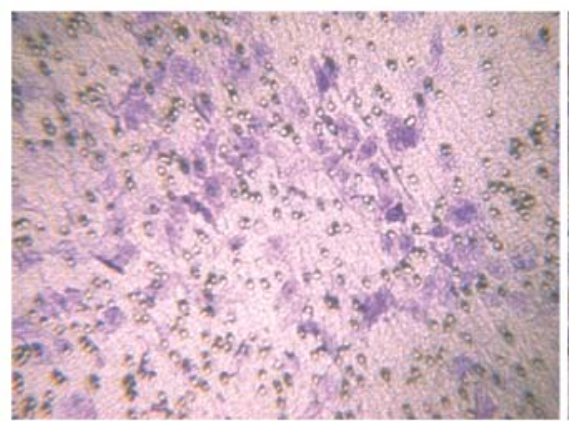

Control

B

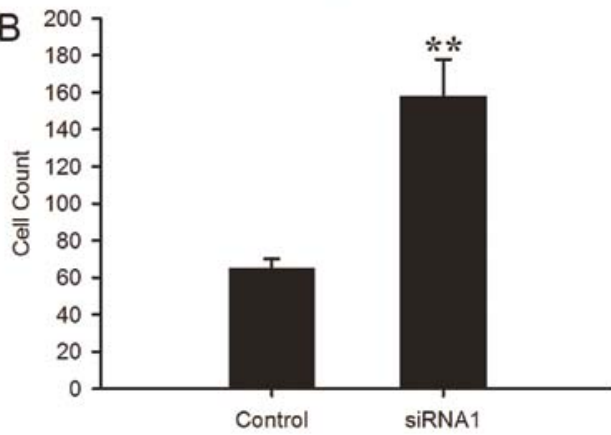

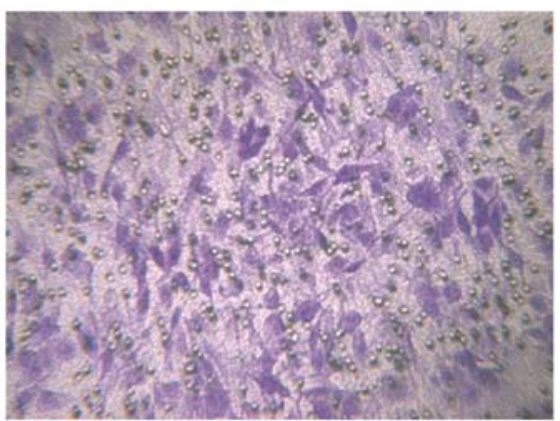

SiRNA1

C

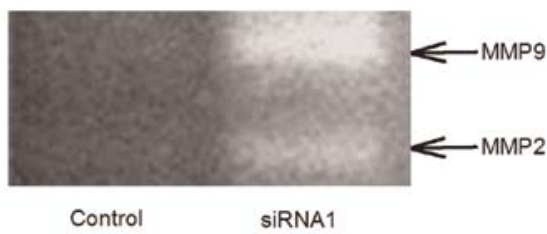

Figure 4. Downregulation of LRIG1 increases the invasive capability of the GL15 cells. (A) Invasive ability was measured by the Transwell invasion chamber assay; 2 representative microscopic fields are shown. (B) The number of invading cells was counted under a microscope (magnification, $\mathrm{x} 200$ ) in 5 predetermined fields, and the results from 3 separate chambers were then averaged and shown as the means \pm SD $\left({ }^{* *} \mathrm{P}<0.01\right)$. (C) Effects of gelatinolytic activity were measured by gelatin zymography. The gelatinolytic activity of MMP-2 and MMP-9 in LRIG1-siRNA cells was increased compared to the control.

with EGF, the phosphorylation of EGFR in the LRIG1-siRNA1 cells was notably increased particularly under the 5-min stimulation (Fig. 5B). The phosphorylated Akt level was also observed to be increased in the LRIG1-siRNA1 group for all three stimulation points, whereas, the ERK phosphorylation in the LRIG1-siRNA1 group was not significantly different from the control group. Similar to the results of the treatment with EGF, LRIG1-siRNA1 cells cultured in complete medium for $48 \mathrm{~h}$ after synchronization demonstrated a markedly increased phosphorylated Akt level and no significant difference in the expression of ERK phosphorylation (Fig. 5C). This data clearly indicated that the EGFR-mediated PI3K/Akt pathway was dominantly activated by LRIG1 knockdown. In addition, the oncogene-c-Myc, involved in cell proliferation and cell cycle regulation demonstrated an increased expression in siRNA1 cells (Fig. 5C).

\section{Discussion}

In the present study, we successfully established LRIG1 knockdown glioblastoma cells, in which the expression of LRIG1 was specifically and effectively inhibited by siRNA. The downregulation of LRIG1 markedly enhanced the malignant properties of glioblastoma cells, such as increased proliferative and invasive capabilities and a decreased apoptotic rate. To the best of our knowledge, for the first time, we demonstrated that LRIG1 silencing promoted the aggressive capabilities of glioblastoma cells by the activation of EGFR/AKT/c-Myc.

Glioblastoma multiforme (GBM) is the most common and devastating malignant tumor in the brain. Traditional therapeutic modalities are ultimately ineffective in curing this cancer, due to a diffusive infiltrative growth pattern, which contributes to the difficulty of achieving complete tumor resection and to the radio-resistance of glioma cells (22). Based on an ever-increasing understanding of several key signaling pathways involved in growth, proliferation, survival and apoptosis, it is critical to explore novel therapeutic strategies that target these pathways to improve the treatment of malignant glioma in the future. The overexpression of EGFR is one of the most frequent signaling mutations in GBM. The average amplification rate for EGFR in GBM is $\sim 35 \%$ (23), suggesting that inhibiting EGFR may be a possible therapeutic strategy for GBM treatment. The overexpression of EGFR induces the expression of LRIG1, which, in turn, interacts with EGFR and attenuates the EGFR-mediated signaling pathway (11). However, the exact role of LRIG1 and the underlying mechanism of the interaction of LRIG1 with EGFR in the tumorigenesis and development of glioblastoma remain unknown.

LRIG1-siRNA cells exhibited higher proliferative ability compared with the negative control cells. Consistent with a previous report that cell cycle progression was involved in the process of tumor growth (24), our study revealed a profound effect of LRIG1-knockdown on cell cycle distribution, evidenced by an accumulation of cells in the G2/M phase. G2/M-arrested cells were observed to express an increased amount of survivin to resist chemotherapy (25), indicating that the downregulation of LRIG1 in glioblastoma cells may enhance the capability of chemotherapy resistance. Moreover, the apoptosis rate confirmed by flow cytometry was notably decreased in LRIG1-knockdown cells. From these results, we suggest that the downregulation of LRIG1 promotes cell growth and survival of glioblastoma cells by increasing the proliferative and anti-apoptotic capabilities. 
A

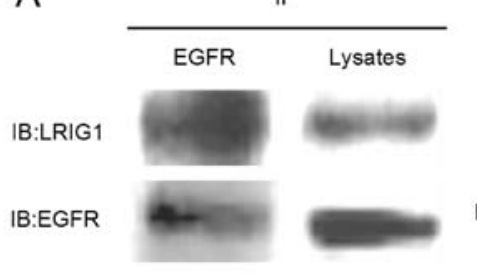

C

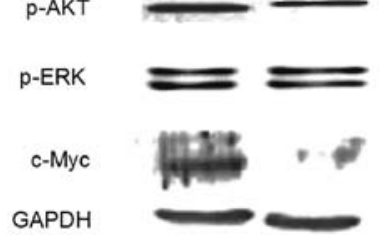

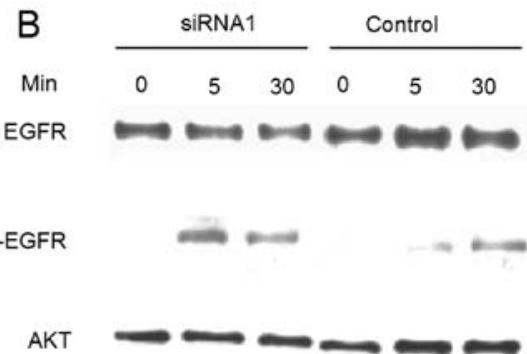

p-AKT

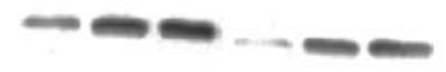

ERK

p-ERK

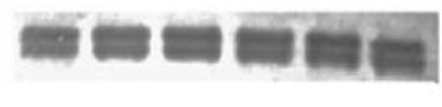

Figure 5. Downregulation of LRIG1 enhances the activation of EGFR, AKT and c-Myc. (A) GL15 cells were cultured in complete medium for 48 h after synchronization. Cell lysates were immunoprecipitated with anti-EGFR antibody and blotted with LRIG1 and EGFR antibodies. (B) After synchronization for $24 \mathrm{~h}$, LRIG1-siRNA1 and the control cells were stimulated with EGF (100 ng/ml) in serum-free medium for the times as indicated. The expression levels of the target proteins were analyzed by western blotting. (C) LRIG1-siRNA1 and the control cells were cultured in normal complete medium for $48 \mathrm{~h}$ after synchronization and cell lysates were detected by western blotting for the levels of the indicated proteins.

Besides uncontrolled cell growth, cell invasion through white matter tracts is also recognized as a hallmark of glioma (26). Adhesion to the extracellular matrix (ECM) and degradation of the ECM are two important steps in tumor cell invasion. Aiming at these processes, we used the ECM-coated Transwell chamber assay to evaluate the effect of LRIG1 knockdown on the invasive ability of GL15 cells. The silencing of LRIG1 in GL15 cells promoted the cell invasive activity in vitro, indicating that LRIG1 may inhibit the invasive capability of glioblastoma cells. A crucial role in the process of ECM degradation is attributed to matrix metalloproteinases (MMPs) especially MMP-2 and MMP-9, which are considered to be suitable predictors of glioma cell invasion and demonstrate a positive correlation with the histopathological malignant grade of glioma $(27,28)$. Gelatin zymography, which was used to determine the activities of MMP-2 and MMP-9, demonstrated that levels of both proteins were increased following LRIG1 knockdown of the GL15 cells, suggesting that LRIG1 knockdown enhanced the invasive capability of GL15 cells by increasing MMP-2 and MMP-9 expression.

To further explore the potential mechanisms promoting aggressive behaviors mediated by LRIG1 knockdown, we evaluated the active state of EGFR and its downstream signaling proteins including AKT and MAPK. As mentioned above, EGFR was overexpressed in multiple GBM cases and played a significant role in regulating other intracellular signaling pathways including PI3K/Akt and RAS/MAPK, contributing to cell survival, proliferation, invasion and angiogenesis $(29,30)$.

A previous study with other cell lines demonstrated that the upregulation of LRIG1 promoted ubiquitylation and degradation of EGFR by receptor combination with leucine-rich repeat (LRR) as well as immunoglobulin-like (Ig) domains of LRIG1 protein (11). Consistent with previous studies that LRIG1 may form a protein complex with EGFR in non-glioma cell lines $(11,31)$ our study confirmed that endogenous LRIG1 interacted with endogenous EGFR and formed a protein complex in glioblastoma cells. The downregulation of LRIG1 notably increased the activation of EGFR, which was consistent with our previous report that LRIG1 suppressed glioma cell growth by inhibiting EGFR (18). The increased activation of EGFR induced the activation of the downstream signaling pathway molecule Akt, demonstrating that the signaling pathway of PI3K/Akt was enhanced, contributing to the increased proliferative, anti-apoptotic and invasive capabilities of LRIG1-siRNA GL15 cells. However, LRIG1 knockdown in GL15 cells revealed no significant effect on ERK activation. Therefore, we propose that the EGFR-mediated PI3K/Akt pathway was dominantly activated by LRIG1 knockdown, at least in the GL15 cell line.

In addition to the two crucial signaling molecules, we also focused on c-Myc, a proto-oncogene which exhibits a positive correlation with the malignant grade of tumors and contributes to the deregulated proliferation and survival of glioma cells and stem cell self-renewal $(32,33)$. LRIG1 maintains stem cells in quiescence by EGFR downregulation (34), suggesting a potential cross-network between c-Myc and LRIG1 in glioma cells. c-Myc is also an important factor in cell proliferation by cell cycle progression from the G1 to the S phase (35). In our study, we observed that c-Myc expression was markedly increased in LRIG1-siRNA cells, which may partly explain the G2/M arrest of LRIG1 knockdown in the GL15 cells.

To the best of our knowledge, we demonstrated for the first time that the downregulation of LRIG1 promoted the aggressive properties of glioblastoma cells including proliferative, anti-apoptotic and invasive capabilities via enhancing the activation of EGFR/Akt/c-Myc. These results provide profound evidence for the proposal that LRIG1 acts as a tumor suppressor gene in glioblastoma cells. Further investigation 
regarding the underlying mechanisms and biological effects of LRIG1 knockdown in vivo is warranted and the applicability of amplifying LRIG1 in glioblastoma therapy deserves further investigation.

The limitation of this research was that this study was performed in vitro and only focused on one glioblastoma cell line. Further experiments are required to confirm our results using other cell lines in vitro and animal models in vivo.

\section{Acknowledgements}

This study was supported by National Natural and Science Foundation of China (No. 81001116) and the National Clinical Key Specialty Construction Project. Professor Håkan Hedman is acknowledged for kindly providing the GL15 cell line.

\section{References}

1. Stupp R, Mason WP, van den Bent MJ, et al: Radiotherapy plus concomitant and adjuvant temozolomide for glioblastoma. $\mathrm{N}$ Engl J Med 352: 987-996, 2005.

2. Wang B, Han L, Chen R, et al: Downregulation of LRIG2 expression by RNA interference inhibits glioblastoma cell (GL15) growth, causes cell cycle redistribution, increases cell apoptosis and enhances cell adhesion and invasion in vitro. Cancer Biol Ther 8: 1018-1023, 2009.

3. Guo D, Holmlund C, Henriksson R and Hedman H: The LRIG gene family has three vertebrate paralogs widely expressed in human and mouse tissues and a homolog in Ascidiacea. Genomics 84: 157-165, 2004.

4. Nilsson J, Vallbo C, Guo D, et al: Cloning, characterization, and expression of human LIG1. Biochem Biophys Res Commun 284 $1155-1161,2001$.

5. Ishii $\mathrm{H}$ and Furukawa $\mathrm{Y}$ : Alterations of common chromosome fragile sites in hematopoietic malignancies. Int J Hematol 79: 238-242, 2004.

6. Martinez A, Walker RA, Shaw JA, Dearing SJ, Maher ER and Latif F: Chromosome 3p allele loss in early invasive breast cancer: detailed mapping and association with clinicopathological features. Mol Pathol 54: 300-306, 2001.

7. Hedman H, Nilsson J, Guo D and Henriksson R: Is LRIG1 a tumour suppressor gene at chromosome 3p14.3? Acta Oncol 41: $352-354,2002$

8. Thomasson M, Hedman H, Guo D, Ljungberg B and Henriksson R: LRIG1 and epidermal growth factor receptor in renal cell carcinoma: a quantitative RT-PCR and immunohistochemical analysis. Br J Cancer 89: 1285-1289, 2003.

9. Yang WM, Yan ZJ, Ye ZQ and Guo DS: LRIG1, a candidate tumour-suppressor gene in human bladder cancer cell line BIU87. BJU Int 98: 898-902, 2006.

10. Tanemura A, Nagasawa T, Inui S and Itami S: LRIG-1 provides a novel prognostic predictor in squamous cell carcinoma of the skin: immunohistochemical analysis for 38 cases. Dermatol Surg 31: 423-430, 2005

11. Gur G, Rubin C, Katz M, et al: LRIG1 restricts growth factor signaling by enhancing receptor ubiquitylation and degradation. EMBO J 23: 3270-3281, 2004.

12. Lindstrom AK, Ekman K, Stendahl U, et al: LRIG1 and squamous epithelial uterine cervical cancer: correlation to prognosis, other tumor markers, sex steroid hormones, and smoking. Int J Gynecol Cancer 18: 312-317, 2008.

13. Miller JK, Shattuck DL, Ingalla EQ, et al: Suppression of the negative regulator LRIG1 contributes to ErbB2 overexpression in breast cancer. Cancer Res 68: 8286-8294, 2008.
14. Li F, Ye ZQ, Guo DS and Yang WM: Suppression of bladder cancer cell tumorigenicity in an athymic mouse model by adenoviral vector-mediated transfer of LRIG1. Oncol Rep 26: 439-446, 2011.

15. Welsh JB, Sapinoso LM, Su AI, et al: Analysis of gene expression identifies candidate markers and pharmacological targets in prostate cancer. Cancer Res 61: 5974-5978, 2001.

16. Lapointe J, Li C, Higgins JP, et al: Gene expression profiling identifies clinically relevant subtypes of prostate cancer. Proc Natl Acad Sci USA 101: 811-816, 2004.

17. Hedman $\mathrm{H}$ and Henriksson R: LRIG inhibitors of growth factor signalling - double-edged swords in human cancer? Eur J Cancer 43: 676-682, 2007

18. Ye F, Gao Q, Xu T, et al: Upregulation of LRIG1 suppresses malignant glioma cell growth by attenuating EGFR activity. J Neurooncol 94: 183-194, 2009.

19. Guo D, Nilsson J, Haapasalo H, et al: Perinuclear leucine-rich repeats and immunoglobulin-like domain proteins (LRIG1-3) as prognostic indicators in astrocytic tumors. Acta Neuropathol 111: 238-246, 2006.

20. Yuan B, Latek R, Hossbach M, Tuschl T and Lewitter F: siRNA Selection Server: an automated siRNA oligonucleotide prediction server. Nucleic Acids Res 32: W130-W134, 2004.

21. Tuschl T: Expanding small RNA interference. Nat Biotechnol 20: 446-448, 2002.

22. Golding SE, Morgan RN, Adams BR, Hawkins AJ, Povirk LF and Valerie K: Pro-survival AKT and ERK signaling from EGFR and mutant EGFRvIII enhances DNA double-strand break repair in human glioma cells. Cancer Biol Ther 8: 730-738, 2009.

23. Rao SK, Edwards J, Joshi AD, Siu IM and Riggins GJ: A survey of glioblastoma genomic amplifications and deletions. J Neurooncol 96: 169-179,2010.

24. Yeh KY, Chang JW, Li YY, Wang CH and Wang HM: Tumor growth inhibition of metastatic nasopharyngeal carcinoma cell lines by low dose of arsenic trioxide via alteration of cell cycle progression and induction of apoptosis. Head Neck 33: 734-742, 2011.

25. Chandele A, Prasad V, Jagtap JC, Shukla R and Shastry PR: Upregulation of survivin in G2/M cells and inhibition of caspase 9 activity enhances resistance in staurosporine-induced apoptosis. Neoplasia 6: 29-40, 2004.

26. Tate MC and Aghi MK: Biology of angiogenesis and invasion in glioma. Neurotherapeutics 6: 447-457, 2009.

27. Wild-Bode C, Weller M and Wick W: Molecular determinants of glioma cell migration and invasion. J Neurosurg 94: 978-984, 2001.

28. Rao JS: Molecular mechanisms of glioma invasiveness: the role of proteases. Nat Rev Cancer 3: 489-501, 2003.

29. Kapoor GS and O'Rourke DM: Receptor tyrosine kinase signaling in gliomagenesis: pathobiology and therapeutic approaches. Cancer Biol Ther 2: 330-342, 2003.

30. Fu Y, Zhang Q, Kang C, et al: Inhibitory effects of adenovirus mediated Akt1 and PIK3R1 shRNA on the growth of malignant tumor cells in vitro and in vivo. Cancer Biol Ther 8: 1002-1009, 2009.

31. Laederich MB, Funes-Duran M, Yen L, et al: The leucine-rich repeat protein LRIG1 is a negative regulator of ErbB family receptor tyrosine kinases. J Biol Chem 279: 47050-47056, 2004.

32. Shindo H, Tani E, Matsumuto T, Hashimoto T and Furuyama J: Stabilization of c-myc protein in human glioma cells. Acta Neuropathol 86: 345-352, 1993.

33. Wang J, Wang H, Li Z, et al: c-Myc is required for maintenance of glioma cancer stem cells. PLoS One 3: e3769, 2008.

34. Jensen KB and Watt FM: Single-cell expression profiling of human epidermal stem and transit-amplifying cells: LRIG1 is a regulator of stem cell quiescence. Proc Natl Acad Sci USA 103: 11958-11963, 2006.

35. Dang CV: c-Myc target genes involved in cell growth, apoptosis, and metabolism. Mol Cell Biol 19: 1-11, 1999. 